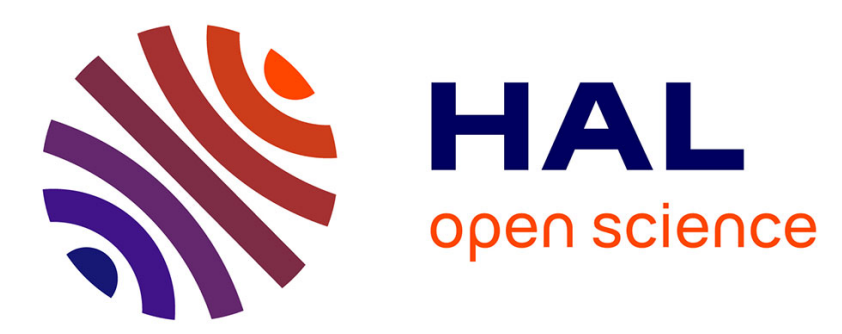

\title{
Two-Phase Multiscale FEM Modelling of Macrosegregation Formation in Steel Slabs
}

Michel Bellet

\section{To cite this version:}

Michel Bellet. Two-Phase Multiscale FEM Modelling of Macrosegregation Formation in Steel Slabs. Numiform 2007, 9th International Conference on Numerical Methods in Industrial Forming Processes, Jun 2007, Porto, Portugal. p. 1369-1374, 10.1063/1.2741000 . hal-00576593

HAL Id: hal-00576593

https://hal-mines-paristech.archives-ouvertes.fr/hal-00576593

Submitted on 14 Mar 2011

HAL is a multi-disciplinary open access archive for the deposit and dissemination of scientific research documents, whether they are published or not. The documents may come from teaching and research institutions in France or abroad, or from public or private research centers.
L'archive ouverte pluridisciplinaire HAL, est destinée au dépôt et à la diffusion de documents scientifiques de niveau recherche, publiés ou non, émanant des établissements d'enseignement et de recherche français ou étrangers, des laboratoires publics ou privés. 


\title{
Two-Phase Multiscale FEM Modelling of Macrosegregation Formation in Steel Slabs
}

\author{
Michel Bellet \\ Ecole des Mines de Paris, Centre de Mise en Forme des Matériaux (CEMEF), UMR CNRS 7635, BP 207, 06904 \\ Sophia Antipolis, France. michel.bellet@ensmp.fr
}

\begin{abstract}
The conservation equations of a coupled thermomechanical - solute transport model to be applied in mushy zones are first recalled. Then the numerical implementation is discussed, in the context of steel continuous casting modeling. Application to the study of the end of solidification is then considered and the effects of soft reduction process are discussed.
\end{abstract}

Keywords: solidification, mushy zone, 2-phase, thermomechanics, continuous casting, soft reduction. PACS:

\section{INTRODUCTION}

The understanding and control of central macrosegregation in steel continuous casting (CC) is still - after more than 35 years of process development - a major challenge for industry, especially in view of new high strength grades with higher concentrations of alloy elements. In the context of $\mathrm{CC}$, Miyazawa \& Schwerdtfeger [1], and Lesoult \& Sella [2] have demonstrated that macro-segregation in this case highly depends on the deformation of the coherent solid phase in the mushy zone (induced by the bulging between rolls). Following those pioneer works, Kajitani et al. [3] proposed a numerical model, but doing strong assumptions on the solid phase motion. In previous papers $[4,5]$, we have derived a multiscale coupled model for thermomechanical-solute interaction in mushy zones, using spatial averaging on a representative elementary volume of the mushy material. The governing equations of this model are briefly reminded here. Then, its application to the context of CC-modeling is discussed, especially the concurrent modeling of the mushy core and the solid crust of the slab. Finally, an application is presented, including a discussion of the results obtained with and without soft reduction process.

\section{MACROSCOPIC TWO-PHASE MODEL}

\section{Governing Equations. Multiscale Approach by Spatial Averaging}

At the microscopic scale, inside each phase (liquid and solid), the thermo-mechanical evolution is assumed to be governed by the usual mass, momentum energy and solute balances. In this work, the balance equations of the mixture, at the macroscopic scale of a representative elementary volume (REV), are obtained using the spatial averaging method (see $[6,7]$ for details and notations). The solidifying alloy in the mushy state is considered as a saturated two-phase medium $\left(g_{s}+g_{l}=1\right)$. Applying the averaging process to microscopic balance equations in each phase $k(k=s, l)$, the following equations (1) are obtained, in which $\rho$ denotes the density, $v$ the velocity field, $\boldsymbol{\sigma}$ the stress tensor, $\boldsymbol{g}$ the gravity vector, $h$ the enthalpy per unit of mass, $\boldsymbol{q}$ the heat flow vector, $c$ the volume concentration, $\boldsymbol{j}$ the solute flow vector. The terms $\Gamma, \boldsymbol{M}, Q$ and $J$ are associated with the exchanges of mass, momentum, energy and solute, respectively, between the two phases. 
Solute

$$
\frac{\partial}{\partial t}\left(g_{k} c_{k}\right)+\nabla \cdot\left(g_{k} c_{k} \boldsymbol{v}_{k}\right)+\nabla \cdot\left\langle\boldsymbol{j}^{k}\right\rangle=J_{k}
$$

\section{Hypotheses and Resulting Macroscopic Equations}

- The assumption of local thermal equilibrium holds within the REV: $T_{s}=T_{l}=T$.

- The solid and liquid phases have constant (but different) densities, in the solidification interval. Solidification shrinkage is then accounted for.

- At the microscopic scale, the liquid metal is considered as an incompressible Newtonian fluid.

- At the microscopic scale, the solid phase is considered as a non-Newtonian generalized fluid, obeying a Norton-Hoff constitutive equation (degree $m$ power-law).

- At the macroscopic scale, a Fick's law is assumed for diffusion in the liquid, while diffusion is neglected in the solid.

- The momentum interaction between solid and liquid phases obeys an isotropic Darcy law.

Given those hypotheses, the following set of conservation equations is obtained [5]:

Solid momentum

$$
\nabla \cdot \Sigma^{s}-g_{s} \nabla p_{l}+g_{l}^{2} \mu_{l} \mathrm{~K}^{-1}\left(\boldsymbol{v}_{l}-\boldsymbol{v}_{s}\right)+g_{s} \rho_{s} \boldsymbol{g}=\rho_{s} \frac{\partial}{\partial t}\left(g_{s} \boldsymbol{v}_{s}\right)+\rho_{s} \nabla \cdot\left(g_{s} \boldsymbol{v}_{s} \times \boldsymbol{v}_{s}\right)
$$

Liquid momentum

$$
\nabla \cdot\left\langle\boldsymbol{s}^{l}\right\rangle-g_{l} \nabla p_{l}-g_{l}{ }^{2} \mu_{l} \mathrm{~K}^{-1}\left(\boldsymbol{v}_{l}-\boldsymbol{v}_{s}\right)+g_{l} \rho_{l} \boldsymbol{g}=\rho_{l} \frac{\partial}{\partial t}\left(g_{l} \boldsymbol{v}_{l}\right)+\rho_{l} \nabla \cdot\left(g_{l} \boldsymbol{v}_{l} \times \boldsymbol{v}_{l}\right)
$$

Mixture mass

$$
\nabla \cdot\left(g_{s} \boldsymbol{v}_{s}\right)+\frac{\rho_{l}}{\rho_{s}} \nabla \cdot\left(g_{l} \boldsymbol{v}_{l}\right)=\frac{\rho_{s}-\rho_{l}}{\rho_{s}} \frac{\partial g_{s}}{\partial t}
$$

Mixture energy

$$
\langle\rho\rangle \frac{\partial\langle h\rangle}{\partial t}+\nabla\langle h\rangle \cdot\langle\rho v\rangle+L \nabla \cdot\left(f_{s} f_{l}\langle\rho\rangle\left(\boldsymbol{v}_{l}-\boldsymbol{v}_{s}\right)\right)-\nabla \cdot(\langle\lambda\rangle \nabla T)=0
$$

Mixture solute

$$
\frac{\partial\langle c\rangle}{\partial t}+\nabla \cdot\left(\langle c\rangle v_{s}\right)+\nabla \cdot\left(g_{l} c_{l}\left(v_{l}-v_{s}\right)\right)-\nabla \cdot\left(g_{l} D_{l} \nabla c_{l}\right)=0
$$

In those equations, the Darcy terms appear clearly, the permeability $\mathrm{K}$ deriving directly from the liquid fraction and a predefined secondary dendrite arm spacing (Carman-Kozeny model).

Regarding constitutive models, like Ganesan \& Poirier [8] and Rappaz et al. [7], the average deviatoric stress tensor in the liquid phase is expressed by:

$$
\begin{aligned}
\Sigma^{l}=\left\langle\boldsymbol{s}^{l}\right\rangle & =2 \mu_{l} g_{l} \operatorname{dev}\left(\dot{\boldsymbol{\varepsilon}}\left(\boldsymbol{v}_{l}\right)\right) \\
& =2 \mu_{l} g_{l}\left(\dot{\boldsymbol{\varepsilon}}\left(\boldsymbol{v}_{l}\right)-\frac{1}{3} \operatorname{tr}\left(\dot{\boldsymbol{\varepsilon}}\left(\boldsymbol{v}_{l}\right)\right)\right)
\end{aligned}
$$

For solid, the effective macroscopic stress tensor $\Sigma^{s}=\langle\boldsymbol{\sigma}\rangle+p_{l} \boldsymbol{I}$ can be shown to be a degree $m$ homogeneous function with respect to the strain rate tensor $\langle\dot{\boldsymbol{\varepsilon}}\rangle^{S}=\dot{\boldsymbol{\varepsilon}}\left(\boldsymbol{v}_{S}\right)$. Adopting a compressible viscoplastic potential, we have [5]:

$$
\Sigma^{s}=3 K\left(\sqrt{3}\langle\dot{\varepsilon}\rangle_{e q}^{s}\right)^{m-1}\left(\frac{1}{A}\langle\dot{\boldsymbol{\varepsilon}}\rangle^{S}+\left(\frac{1}{9 B}-\frac{1}{3 A}\right) \operatorname{tr}\langle\dot{\boldsymbol{\varepsilon}}\rangle^{s} \boldsymbol{I}\right)
$$

in which the two rheological functions $A$ and $B$ depend on the solid volume fraction, according to various models and the equivalent strain rate is expressed as:

$$
\langle\dot{\boldsymbol{\varepsilon}}\rangle_{e q}^{s^{2}}=\frac{1}{A}\langle\dot{\boldsymbol{\varepsilon}}\rangle^{s}:\langle\dot{\boldsymbol{\varepsilon}}\rangle^{s}+\left(\frac{1}{9 B}-\frac{1}{3 A}\right)\left(\operatorname{tr}\langle\dot{\boldsymbol{\varepsilon}}\rangle^{s}\right)^{2}
$$

Note also that in energy and solute equations (2d) and (2e), there are specific terms arising from the compression or dilatation of the solid phase.

\section{Microsegregation Model (Solute Exchange at Micro-Scale)}

The solute exchange between the solid and liquid phase is governed by interfacial phenomena occurring 
at the scale of dendrite arms. This is a non-equilibrium process involving complex physics. However, several simplifying hypotheses are allowed in the context of macrosegregation studies. Besides the uniformity of temperature within the REV, both phases are assumed to be at thermodynamic equilibrium at their interface, according to the alloy phase diagram. Then, the interfacial alloy concentrations satisfy:

$$
c_{s}^{*}=k(T) c_{l}^{*} \text { and } c_{l}^{*}=\psi(T)
$$

where the thermo-dependent partition coefficient $k$, as well as the equation of the liquidus line $\psi(T)$, can be determined from the phase diagram. In this study, both $k$ and the slope of the liquidus line $\partial \psi / \partial T$ are assumed constant. Further, we assume a perfect diffusion of solute in the liquid and in the solid phase at the microscopic scale, so that we obtain:

$$
\begin{aligned}
& \langle c\rangle=\left(g_{l}+\left(1-g_{l}\right) k\right) c_{l} \\
& T=T_{m}+\left(\frac{\partial \psi}{\partial T}\right)^{-1} c_{l}
\end{aligned}
$$

where $T_{m}$ is the fusion temperature of the pure substance and $L$ is the specific latent heat. Keeping in mind the expression of the average specific enthalpy,

$$
\langle h\rangle=\int_{T_{0}}^{T} c_{p}(\tau) d \tau+\frac{g_{l} \rho_{l}}{g_{l} \rho_{l}+\left(1-g_{l}\right) \rho_{s}} L
$$

it can be seen that given $\langle c\rangle$ and $\langle h\rangle,(7)$ and (8) form a non-linear set of three equations from which the values $g_{l}, T, c_{l}, c_{s}$ can be calculated.

\section{Numerical Implementation}

The above equations are solved by a twodimensional finite element method. Hence they are treated in a weak (i.e. integral) form. For CC application, inertia terms are neglected in solid and liquid momentum equations, because of low solid and liquid velocities.

\section{Weak Form of the Mechanical Problem}

As shown in next section, the calculation domain encompasses fully solidified regions and coherent mushy zones. Therefore, during the finite element assembly procedure, we treat differently fully solid and mushy finite elements (according to the temperature at element centre).

In the case of a mushy element, its contributions to the residues to be assembled are as indicated in (9) for conservation of solid momentum, liquid momentum and mass $\left(\boldsymbol{u}^{*}, \boldsymbol{v}^{*}\right.$ and $p^{*}$ being test functions).

In the case of a fully solid element, the expressions of those residue contributions are as indicated in (10).

\section{Mushy Element:}

$$
\begin{aligned}
& \boldsymbol{R}_{\text {sol }}^{\text {elt }}=\int_{\Omega^{\text {elt }}} \Sigma^{s}: \nabla \boldsymbol{u}^{*}-\int_{\Omega^{\text {elt }}} p_{l} \nabla \cdot\left(g_{s} \boldsymbol{u}^{*}\right)-\int_{\Omega^{\text {elt }}} g_{l}^{2} \mu_{l} \mathrm{~K}^{-1}\left(\boldsymbol{v}_{l}-\boldsymbol{v}_{s}\right) \cdot \boldsymbol{u}^{*}-\int_{\partial \Omega^{\text {elt }}}\left\langle\boldsymbol{\sigma}^{s}\right\rangle \boldsymbol{n} \cdot \boldsymbol{u}^{*}-\int_{\Omega^{\text {elt }}} g_{s} \rho_{s} \boldsymbol{g} \cdot \boldsymbol{u}^{*} \\
& \boldsymbol{R}_{\text {liq }}^{\text {elt }}=\int_{\Omega^{\text {elt }}}\left\langle\boldsymbol{s}^{l}\right\rangle: \nabla \boldsymbol{v}^{*}-\int_{\Omega^{\text {elt }}} p_{l} \nabla \cdot\left(g_{l} \boldsymbol{v}^{*}\right)+\int_{\Omega^{\text {elt }}} g_{l}{ }^{2} \mu_{l} \mathrm{~K}^{-1}\left(\boldsymbol{v}_{l}-\boldsymbol{v}_{s}\right) \cdot \boldsymbol{v}^{*}-\int_{\partial \Omega^{\text {elt }}}\left\langle\boldsymbol{\sigma}^{l}\right\rangle \boldsymbol{n} \cdot \boldsymbol{v}^{*}-\int_{\Omega^{\text {elt }}} g_{l} \rho_{l} \boldsymbol{g} \cdot \boldsymbol{v}^{*} \\
& \boldsymbol{R}_{\text {mass }}^{\text {elt }}=\int_{\Omega^{\text {elt }}}-p^{*}\left(\nabla \cdot\left(g_{s} \boldsymbol{v}_{s}\right)+\frac{\rho_{l}}{\rho_{s}} \nabla \cdot\left(g_{l} \boldsymbol{v}_{l}\right)-\frac{\partial g_{s}}{\partial t} \frac{\rho_{s}-\rho_{l}}{\rho_{s}}\right)
\end{aligned}
$$

\section{Full Solid Element:}

$$
\begin{aligned}
& \boldsymbol{R}_{\text {sol }}^{\text {elt }}=\int_{\Omega^{\text {elt }}} \boldsymbol{s}: \nabla \boldsymbol{u}^{*}-\int_{\Omega^{\text {elt }}} p \nabla \cdot \boldsymbol{u}^{*}-\int_{\Omega^{\text {elt }}} g_{l}^{2} \mu_{l} \mathrm{~K}^{-1}\left(\boldsymbol{v}_{l}-\boldsymbol{v}_{S}\right) \cdot \boldsymbol{u}^{*}-\int_{\partial \Omega^{\text {elt }}} \boldsymbol{\sigma n} \cdot \boldsymbol{u}^{*}-\int_{\Omega^{\text {elt }}} \rho_{s} \boldsymbol{g} \cdot \boldsymbol{u}^{*} \\
& \boldsymbol{R}_{\text {liq }}^{\text {elt }}=\int_{\Omega^{\text {elt }}} g_{l}^{2} \mu_{l} \mathrm{~K}^{-1}\left(\boldsymbol{v}_{l}-\boldsymbol{v}_{S}\right) \cdot \boldsymbol{v}^{*} \\
& \boldsymbol{R}_{\text {mass }}^{\text {elt }}=\int_{\Omega^{\text {elt }}}-p^{*}\left(\nabla \cdot \boldsymbol{v}_{S}+\frac{1}{\rho_{s}} \frac{\mathrm{d} \rho_{s}}{\mathrm{~d} t}\right)
\end{aligned}
$$

It can be noted that in the fully solid regions the liquid velocity is still present, but has no real physical meaning: it is kept close to the solid velocity field by means of the sole Darcy term in the liquid momentum equation, acting as a penalty term. The deviatoric stress tensor $s$ is expressed as a function of the velocity field by the viscoplastic Norton-Hoff power law. The material is quasi-incompressible (note the difference between (9c) and (10c): only thermal dilatation terms appear in the contribution to mass conservation.

The integrals in (9) and (10) are spatially discretized using $\mathrm{P} 1+/ \mathrm{P} 1+/ \mathrm{P} 1$ triangle elements [5], using bubble-type additional interpolation functions for liquid and solid velocities, the pressure being linear 
and continuous. Their assembly yields a non-linear set of equations to be solved for nodal values of liquid and solid velocities, and pressure ( 5 unknowns per node in 2D). After resolution, the finite element mesh is updated using the solid velocity: for each node $m$,

$$
\boldsymbol{x}_{t+\Delta t}^{m}=\boldsymbol{x}_{t}^{m}+\boldsymbol{v}_{m s h}^{m} \Delta t \quad \text { with } \quad \boldsymbol{v}_{m s h}^{m}=\boldsymbol{v}_{s}^{m}
$$

\section{Weak Forms of Thermal and Solutal Problems}

The partial time derivative of the enthalpy appearing in (2d) is decomposed into a time derivative with respect to the mesh, moving at velocity $\boldsymbol{v}_{s}$, and an advection term (a similar transformation is done in the solute equation). The residues to be assembled are as indicated in (12) and the resulting non-linear equations are solved for the nodal values of the average specific enthalpy $\langle h\rangle$. Similarly, (13) gives the expression of the contributions to the solutal residues.The resulting non-linear equations are solved for the nodal values of the average concentration $\langle c\rangle$. After solidification, those values have null prescribed variations. Integrals in (12) and (13) are discretized using P1 triangles.

$$
\begin{aligned}
& R_{\text {ther }}^{\text {elt }}=\int_{\Omega^{\text {elt }}}\langle\rho\rangle \frac{\partial_{m s h}\langle h\rangle}{\partial t} \varphi+\int_{\Omega^{\text {elt }}} \nabla\langle h\rangle \cdot\left(\langle\rho v\rangle-\langle\rho\rangle \boldsymbol{v}_{s}\right) \varphi+\int_{\Omega^{\text {elt }}}\langle\lambda\rangle \nabla T \cdot \nabla \varphi-\int_{\partial \Omega^{\text {elt }}} \phi(T) \varphi+\int_{\Omega^{\text {elt }}} L \nabla \cdot\left(f_{s} f_{l}\langle\rho\rangle\left(\boldsymbol{v}_{l}-\boldsymbol{v}_{s}\right)\right) \varphi \\
& R_{\text {slte }}^{\text {elt }}=\int_{\Omega^{\text {elt }}} \frac{\partial_{m s h}\langle c\rangle}{\partial t} \varphi+\int_{\Omega^{\text {elt }}}\langle c\rangle \nabla \cdot \boldsymbol{v}_{S} \varphi+\int_{\Omega^{\text {elt }}} \nabla \cdot\left(g_{l} c_{l}\left(\boldsymbol{v}_{l}-\boldsymbol{v}_{S}\right)\right) \varphi-\int_{\partial \Omega^{\text {elt }}} \varphi g_{l} D_{l} \nabla c_{l} \cdot \boldsymbol{n}+\int_{\Omega^{\text {elt }}} \nabla \varphi \cdot g_{l} D_{l} \nabla c_{l}
\end{aligned}
$$

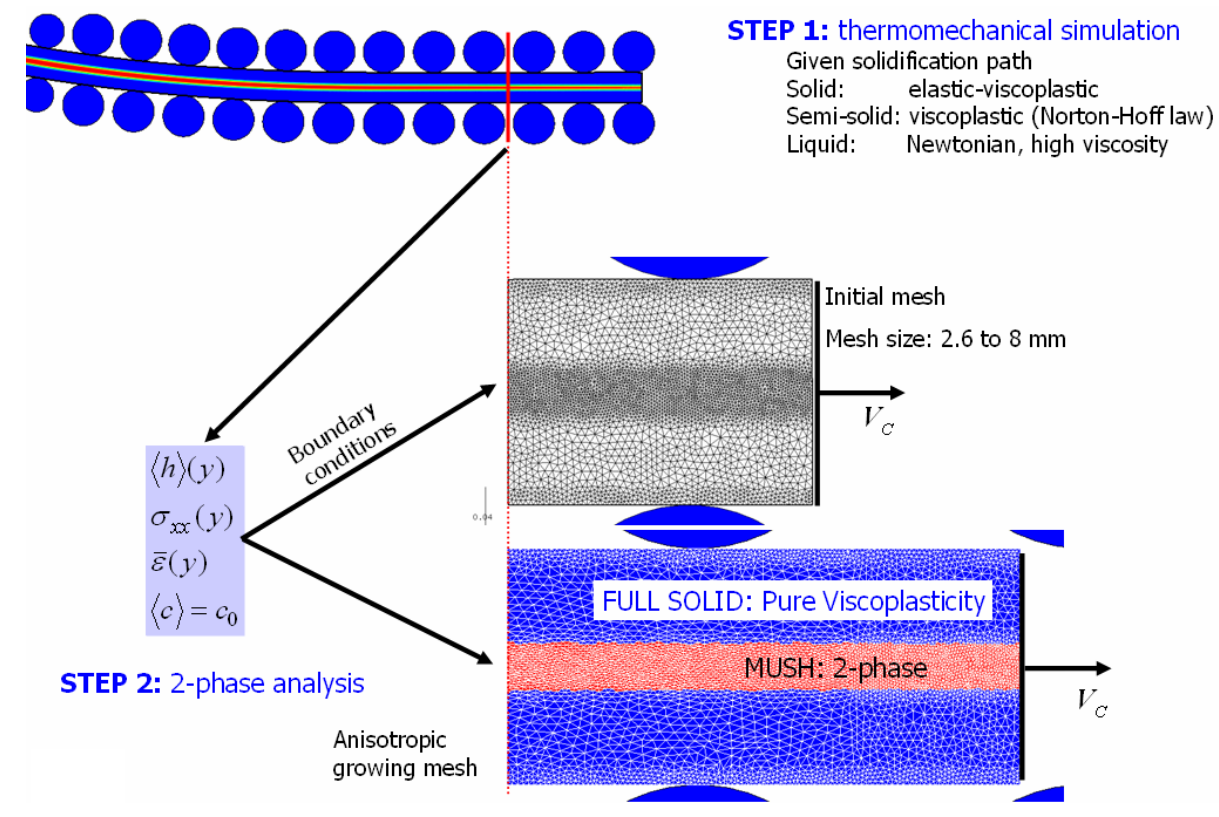

FIGURE 1. Two-step strategy for studying the end of solidification with the present model.

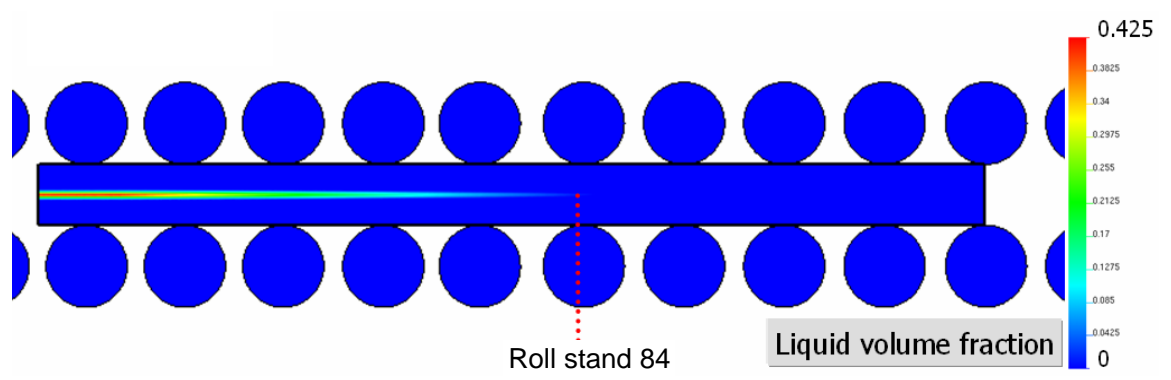

FIGURE 2. Distribution of liquid fraction. Solidification ends $23.5 \mathrm{~m}$ below the meniscus, in roll stand 84 . 
At each time increment, the successive problems are solved in a staggered scheme:

i) energy conservation (global solution);

ii) solute conservation (global solution);

iii) microsegregation (local solution - lever rule at each node);

iv) momentum conservation (liquid and solid) and mass conservation (global solution).

\section{APPLICATION TO STEEL CONTINUOUS CASTING}

The material is modeled as a binary $\mathrm{Fe}-0.18 \mathrm{wt} \% \mathrm{C}$ alloy. The casting velocity is $0.9 \mathrm{~m} / \mathrm{min}$ and the product thickness is $222 \mathrm{~mm}$. In order to apply the two-phase formulation to a coherent mushy zone, we proceed in two steps. In a first step, a simpler thermomechanical analysis is performed, using a model in which the mushy material is considered as a nonNewtonian fluid, without distinction between solid and liquid velocity fields [9]. From this first calculation, we extract the initial and boundary conditions that are applied to a second calculation performed with the present effective 2-phase model. The data transfer is illustrated in Fig. 1.

From step 1 calculation, we can determine at which depth in the machine the mushy core is fully coherent, i.e. when the solid fraction is greater than 0.65 , typically. We initialize then the two-phase calculation starting from this section. The computational mesh grows at the casting speed, starting from a small mesh that also serves as a buffer in the simulation. Along the inlet section of this buffer, the profiles of average enthalpy, normal stress and cumulated plastic strain (in full solid) are imposed. In addition, a uniform nominal solute concentration is assumed, expressing the hypothesis that no segregation has formed yet.

The progress of solidification in the strand is illustrated in Fig. 2. At this stage, the computational domain is $3.5 \mathrm{~m}$ long and contains 29000 nodes.

Two different calculations have been carried out. A first one in which the thickness of the strand is kept constant A second one in which this thickness is slightly reduced, as illustrated schematically in Fig. 3. The squeezing ratio of this so-called "soft reduction" process is $1 \mathrm{~mm} / \mathrm{m}$.

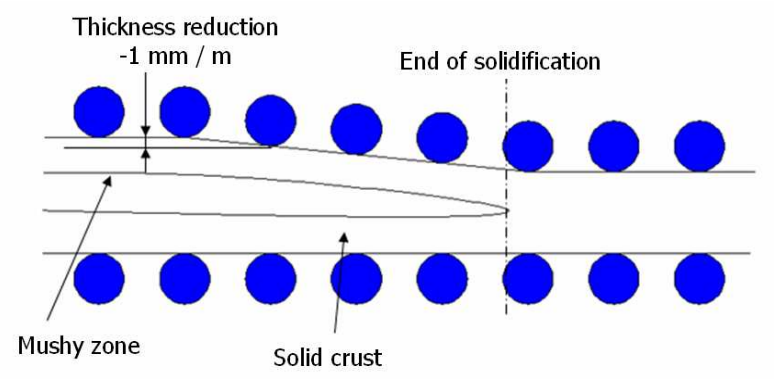

FIGURE 3. Schematic of the soft reduction process studied.

In Fig. 4 and 5 are plotted the axial profiles of liquid fraction and liquid pressure in both cases. It can be seen that the squeezing effect has a minor effect on the advancement of solidification, causing a very slight acceleration of the solidification. The effect is more pronounced on the liquid fraction, which - as expected - is significantly increased in the case of soft reduction. It can be noticed that a constant strand thickness results in large negative values of liquid pressure. In that case (which is not realistic), the shrinkage and dilatation effects are not balanced by any strand reduction: the calculation shows an obvious risk of internal porosity.

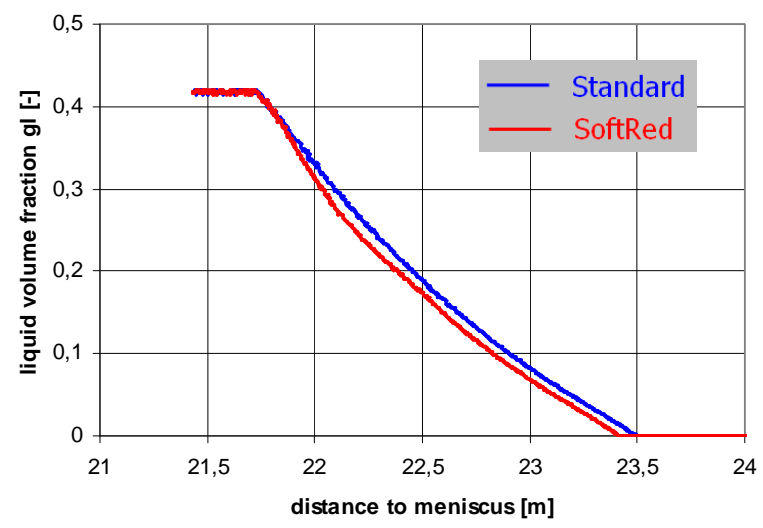

FIGURE 4. Axial distribution of liquid fraction, without and with soft reduction process. 


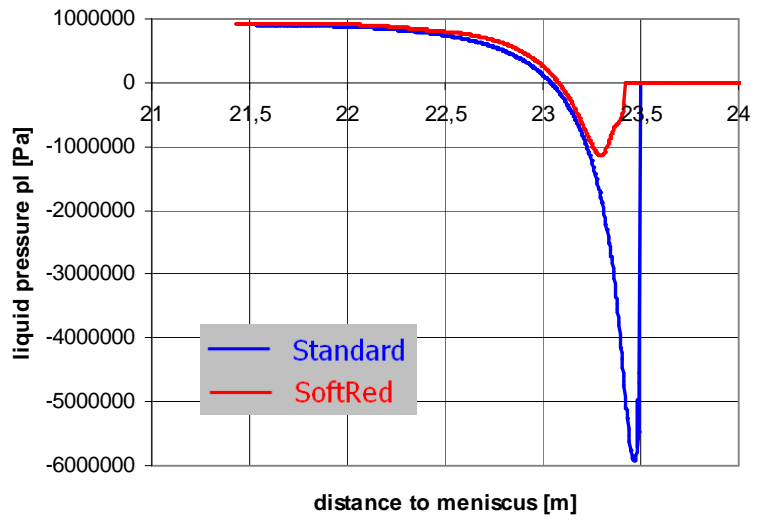

FIGURE 5. Axial distribution of liquid pressure, without and with soft reduction process.

The consequence of soft reduction on macrosegregation is illustrated in Fig. 6. As expected, the squeezing effect produces a decrease in the positive macrosegregation. It can be seen that the maximum relative macrosegregation which is about $0.162 / 0.150=+8 \%$ in the standard process (such a ratio agrees with industrial measurements), is reduced to $0.158 / 0.150=+5 \%$ using soft reduction.

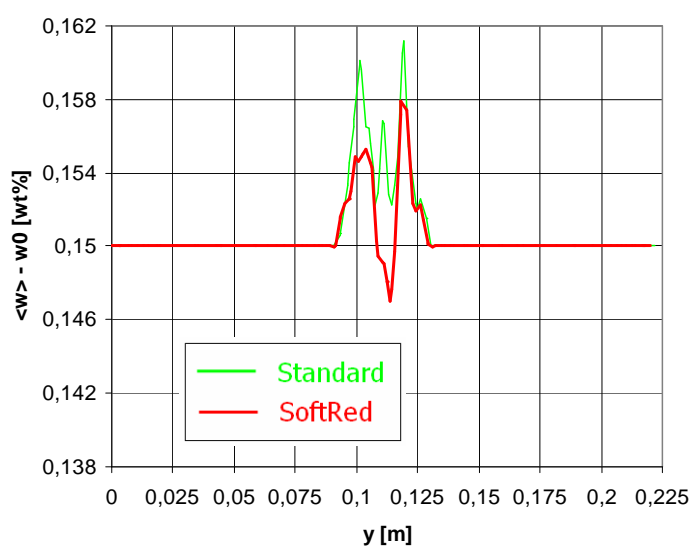

FIGURE 6. Distribution of the average mass concentration of solute $\langle w\rangle$ across slab thickness in stand 86, after complete solidification, with and without soft reduction process.

\section{CONCLUSION}

The coupled macroscopic thermal-mechanicalsolutal conservation equations for a two-phase continuum have been briefly reminded. A twodimensional finite element solution has been proposed and applied to the context of solidification end in slabCC. The qualitative response of the model is quite encouraging. Further improvements, including a multiconstituent microsegregation model and more accurate constitutive parameters should result in a predictive tool to be applied to process design.

\section{ACKNOWLEDGMENTS}

The support of Arcelor Research SA (ArcelorMittal group) is gratefully acknowledged.

\section{REFERENCES}

1. K. Miyazawa, K. Schwerdtfeger, Macrosegregation in continuously cast steel slabs: preliminary theoretical investigation on the effect of steady state bulging, Arch. Eisenhüttenwes. 52 (1981) 415-422.

2. G. Lesoult, S. Sella, Analysis and prevention of centreline segregation during continuous casting of steel related to deformation of the solid phase, Proc. $6^{\text {th }}$ Int. Iron and Steel Congress, Nagoya, Iron and Steel Institute of Japan, vol. 1 (1990) 681-688.

3. T. Kajitani, J.M. Drezet, M. Rappaz, Numerical simulation of deformation induced segregation in continuous casting of steel, Metall. and Mater. Trans. A 32 (2001) 1479-1491.

4. M. Bellet, V.D. Fachinotti, A two-phase twodimensional finite element thermomechanics and macrosegregation model of mushy zone. Application to continuous casting, Proc. MCWASP XI, $11^{\text {th }}$ Int. Conf. on Modeling of Casting, Welding and Advanced Solidification Processes, Opio, France, C.A. Gandin, M. Bellet (eds.), The Minerals, Metals \& Materials Society, Warrendale, Pennsylvania, USA (2006) 169-176

5. V.D. Fachinotti, S. Le Corre, N. Triolet, M. Bobadilla, M. Bellet, Two-phase thermo-mechanical and macrosegregation modelling of binary alloys solidification with emphasis on the secondary cooling stage of steel slab continuous casting processes, Int. J. Num. Meth. Eng. 67 (2006) 1341-1384.

6. J. Ni, C. Beckermann, A volume-averaged two-phase model for transport phenomena during solidification, Metall. Trans. B 22 (1991) 349-361.

7. M. Rappaz, M. Bellet, M. Deville, Numerical modelling in materials science and engineering, Springer Verlag, New York (2003).

8. S. Ganesan, D.R. Poirier, Conservation of mass and momentum for the flow of interdendritic liquid during solidification, Metall. Trans. B 21 (1990) 173-181.

9. M. Bellet, A. Heinrich, A two-dimensional finite element thermomechanical approach to a global stressstrain analysis of steel continuous casting, ISIJ Int. 44 (2004) 1686-1695. 